\title{
Genetic variability of genes involved in DNA repair influence treatment outcome in osteosarcoma
}

\author{
M.J. Wang, Y. Zhu, X.J. Guo and Z.Z. Tian \\ Department of Orthopaedics, Xinxiang Central Hospital, Xinxiang, China \\ Corresponding author: M.J. Wang \\ E-mail: wang_mingjingj@163.com \\ Genet. Mol. Res. 14 (3): 11652-11657 (2015) \\ Received March 3, 2015 \\ Accepted July 1, 2015 \\ Published September 28, 2015 \\ DOI http://dx.doi.org/10.4238/2015.September.28.17
}

ABSTRACT. We conducted a perspective study to investigate the role of ERCC1 (rs11615), ERCC2 (rs13181 and rs1799793), ERCC4 (rs1800067), and ERCC5 ( $\mathrm{rs} 17655$ ) in NER pathway in the prognosis of osteosarcoma patients. In total, 146 osteosarcoma patients were recruited between 2008 and 2013. ERCC1 rs11615, ERCC2 rs13181 and rs1799793, ERCC4 rs1800067, and ERCC5 rs17655 gene polymorphisms were assessed by polymerase chain reaction-restriction fragment length polymorphism assay. By multivariate Cox proportional hazards models, we found that carriers of ERCC1 rs11615 TT genotype showed significantly favorable survival compared to wide-type CC genotype, and the adjusted OR $(95 \% \mathrm{Cl})$ was 0.24 (0.08-0.96). Moreover, we found that subjects with ERCC2 rs1799793 AA genotype were associated with decreased hazards of death in multivariate analysis $(\mathrm{HR}=0.22,95 \% \mathrm{Cl}=0.12-0.93)$. In conclusion, our results suggest that ERCC1 rs 11615 and ERCC2 rs 1799793 may be useful genetic prognostic markers for osteosarcoma in a Chinese population.

Key words: DNA repair genes; Osteosarcoma; Polymorphism; Overall survival 


\section{INTRODUCTION}

Osteosarcoma is derived from mesenchymal tissues and often occurs in the distal femur, proximal tibia, and humeral metaphysis. Osteosarcoma is more common in children and adolescents than in adults, with an annual incidence of about 3/1,000,000 (Picci, 2007; Mirabello et al., 2009; Ottaviani and Jaffe, 2009). Although remarkable progress has been made in surgical treatment, the survival of osteosarcoma remains poor. Moreover, patients with the same TNM stages and treatment may demonstrate varying clinical outcomes, indicating that the progression of osteosarcoma is influenced by multiple factors (Liu et al., 2014; He et al., 2014). Therefore, identifying genetic biomarkers may improve the overall survival of osteosarcoma patients.

DNA repair systems maintain the stability and integrity of the genome, including nucleotide excision repair (NER) (Goode et al., 2002). NER includes damage recognition, damage demarcation and unwinding, damage incision, and new strand ligation, which may influence the effectiveness of therapeutic agents (Nouspikel, 2009). Polymorphisms in NER genes can alter the NER ability by influencing the expression and function of important proteins, thereby altering the survival of individual osteosarcoma patients. Polymorphisms in several NER genes have been studied to determine their relationship with the prognosis of osteosarcoma patients (Bai et al., 2013; Goričar et al., 2015), but the results have been inconsistent. Therefore, we conducted a perspective study to investigate the role of ERCC1 rs11615, ERCC2 rs13181 and rs1799793, ERCC4 rs1800067, and ERCC5 rs17655 in the NER pathway in the prognosis of osteosarcoma patients.

\section{MATERIAL AND METHODS}

A total of 146 osteosarcoma patients were recruited from the Xinxiang Central Hospital between 2008 and 2013. Written informed consent was obtained from participants. Patients who had other malignant tumors or distant metastasis found preoperatively and who had undergone preoperative radiotherapy or chemotherapy were excluded from this study. Medical histories were acquired by questionnaire and the records were computerized.

The Ethical Committee of Xinxiang Central Hospital approved the study protocols, and all participants gave written informed consent.

\section{DNA extraction and genotyping}

The commercially available Qiagen kit (QIAGEN Inc., Hilden, Germany) was used to extract DNA from peripheral blood leukocytes. A polymerase chain reaction-restriction fragment length polymorphism assay was used to assess the ERCC1 rs11615, ERCC2 rs13181 and rs1799793, ERCC4 rs1800067, and ERCC5 rs17655 gene polymorphisms. Polymerase chain reactions were carried out in a Perkin-Elmer 9700 thermocycler (Waltham, MA, USA) with an initial denaturation step of $8 \mathrm{~min}$ at $94^{\circ} \mathrm{C}$, followed by 30 cycles at $94^{\circ} \mathrm{C}$ for $30 \mathrm{~s}$, annealing at $60^{\circ} \mathrm{C}$ for 30 $\mathrm{s}$, and extension at $72^{\circ} \mathrm{C}$ for $1 \mathrm{~min}$. The resulting DNA fragments were separated by $3.5 \%$ agarose gel electrophoresis and visualized under UV light after ethidium bromide staining.

\section{Statistical analysis}

SAS (version 8.01; SAS Institute, Cary, NC, USA) was used for statistical analysis. 
Differences between continuous variables are reported as means \pm standard deviation, while those between categorical variables were evaluated using frequencies and percentages. Univariate and multivariate Cox proportional hazard models were performed to estimate the effect of ERCC1 (rs11615), ERCC2 (rs13181 and rs1799793), ERCC4 (rs1800067), and ERCC5 (rs17655) on the survival of osteosarcoma patients, and the results were assessed by adjusted hazards ratios and $95 \%$ confidence intervals. The Kaplan-Meier method was applied to visualize overall survival in different genotype groups. Two-tailed $\mathrm{P}$ values $<0.05$ were considered to be statistically significant.

\section{RESULTS}

This study included 146 patients with osteosarcoma patients with a mean age of $17.6 \pm$ 8.5 years. Baseline demographic characteristics of osteosarcoma patients are shown in Table 1. Of 146 osteosarcoma patients, 85 patients (58.22\%) were in the III-IV tumor stage, 107 (73.29\%) received limb salvage therapy, $102(69.86 \%)$ had tumor at long tubular bones, and $100(68.49 \%)$ showed metastasis.

The relationships between all polymorphisms in NER genes and osteosarcoma survival in different genetic models are summarized in Table 2. Based on multivariate Cox proportional hazard models, carriers of ERCC1 rs11615 TT genotype showed significantly favorable survival than those with the wild-type CC genotype, and the adjusted odds ratio ( $95 \%$ confidence interval) was 0.24 (0.08-0.96). Moreover, we found that subjects with the ERCC2 rs1799793 AA genotype were associated with decreased hazards of death in multivariate analysis (hazard ratio $=0.22$, $95 \%$ confidence interval $=0.12-0.93$ ). According to Kaplan-Meier analysis, the ERCC1 rs11615 TT genotype and ERCC2 rs1799793 AA genotype were associated with longer overall survival when compared with those with the wild-type genotype (Figures 1 and 2).

\begin{tabular}{|c|c|c|}
\hline Characteristics & Frequencies of osteosarcoma patients & $\%$ \\
\hline \multicolumn{3}{|l|}{ Age (years) } \\
\hline$<20$ & 98 & 67.12 \\
\hline$\geq 20$ & 48 & 32.88 \\
\hline \multicolumn{3}{|l|}{ Gender } \\
\hline Male & 94 & 64.38 \\
\hline Female & 52 & 35.62 \\
\hline \multicolumn{3}{|l|}{ Tumor stage } \\
\hline $\mathrm{I}-\mathrm{II}$ & 61 & 41.78 \\
\hline III-IV & 85 & 58.22 \\
\hline \multicolumn{3}{|l|}{ Type of therapy } \\
\hline Amputation & 39 & 26.71 \\
\hline Limb salvage & 107 & 73.29 \\
\hline \multicolumn{3}{|l|}{ Tumor location } \\
\hline Long tubular bones & 102 & 69.86 \\
\hline Axial skeleton & 44 & 30.14 \\
\hline \multicolumn{3}{|l|}{ Metastasis } \\
\hline Yes & 46 & 31.51 \\
\hline No & 100 & 68.49 \\
\hline
\end{tabular}

Genetics and Molecular Research 14 (3): 11652-11657 (2015)～ＯFUNPEC-RP www.funpecrp.com.br 
Table 2. Polymorphisms of NER pathway genes and osteosarcoma survival.

\begin{tabular}{|c|c|c|c|c|c|c|}
\hline Gene & Patients & $\%$ & Deaths & $\%$ & Adjusted HR $(95 \% \mathrm{Cl})^{1}$ & $P$ values \\
\hline \multicolumn{7}{|c|}{ ERCC1 rs11615 } \\
\hline $\mathrm{CC}$ & 60 & 41.10 & 30 & 48.39 & 1.0 (Ref.) & - \\
\hline CT & 68 & 46.58 & 27 & 43.55 & $0.57(0.27-1.21)$ & 0.14 \\
\hline TT & 18 & 12.33 & 5 & 8.06 & $0.24(0.08-0.96)$ & 0.03 \\
\hline \multicolumn{7}{|c|}{ ERCC2 rs13181 } \\
\hline AA & 78 & 53.42 & 36 & 58.06 & & \\
\hline$A C$ & 57 & 39.04 & 22 & 35.48 & $0.72(0.31-1.42)$ & 0.36 \\
\hline $\mathrm{CC}$ & 11 & 7.53 & 4 & 6.45 & $0.53(0.11-2.16)$ & 0.48 \\
\hline \multicolumn{7}{|c|}{ ERCC2 rs1799793 } \\
\hline GG & 91 & 62.33 & 43 & 69.35 & 1.0 (Ref.) & - \\
\hline GA & 44 & 30.14 & 18 & 29.03 & $0.97(0.41-2.14)$ & 0.82 \\
\hline AA & 11 & 7.53 & 6 & 9.68 & $0.22(0.12-0.93)$ & 0.02 \\
\hline \multicolumn{7}{|c|}{ ERCC4 rs1800067 } \\
\hline GG & 119 & 81.51 & 52 & 83.87 & 1.0 (Ref.) & - \\
\hline GA & 16 & 10.96 & 7 & 11.29 & $0.90(0.25-2.65)$ & 0.81 \\
\hline AA & 11 & 7.53 & 3 & 4.84 & $0.45(0.09-2.17)$ & 0.26 \\
\hline \multicolumn{7}{|c|}{ ERCC5 rs17655 } \\
\hline GG & 77 & 52.74 & 34 & 54.84 & 1.0 (Ref.) & - \\
\hline GC & 54 & 36.99 & 24 & 38.71 & $0.85(0.40-1.82)$ & 0.65 \\
\hline CC & 15 & 10.27 & 4 & 6.45 & $0.82(0.21-2.76)$ & 0.72 \\
\hline
\end{tabular}

${ }^{1}$ Adjusted for age, gender, tumor stage, type of therapy, tumor location, and metastasis.

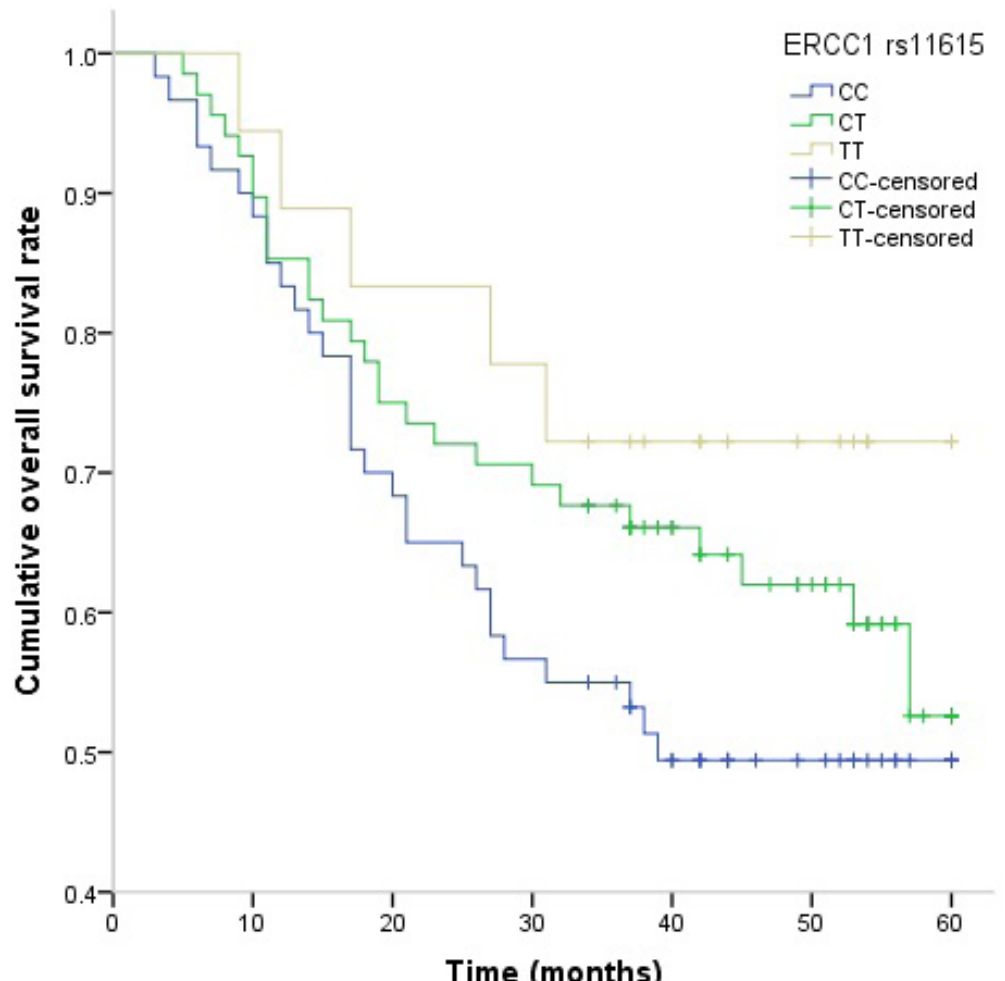

Figure 1. Kaplan-Meier survival curves showing relationship between ERCC1 rs11615 polymorphisms and osteosarcoma patient survival. 


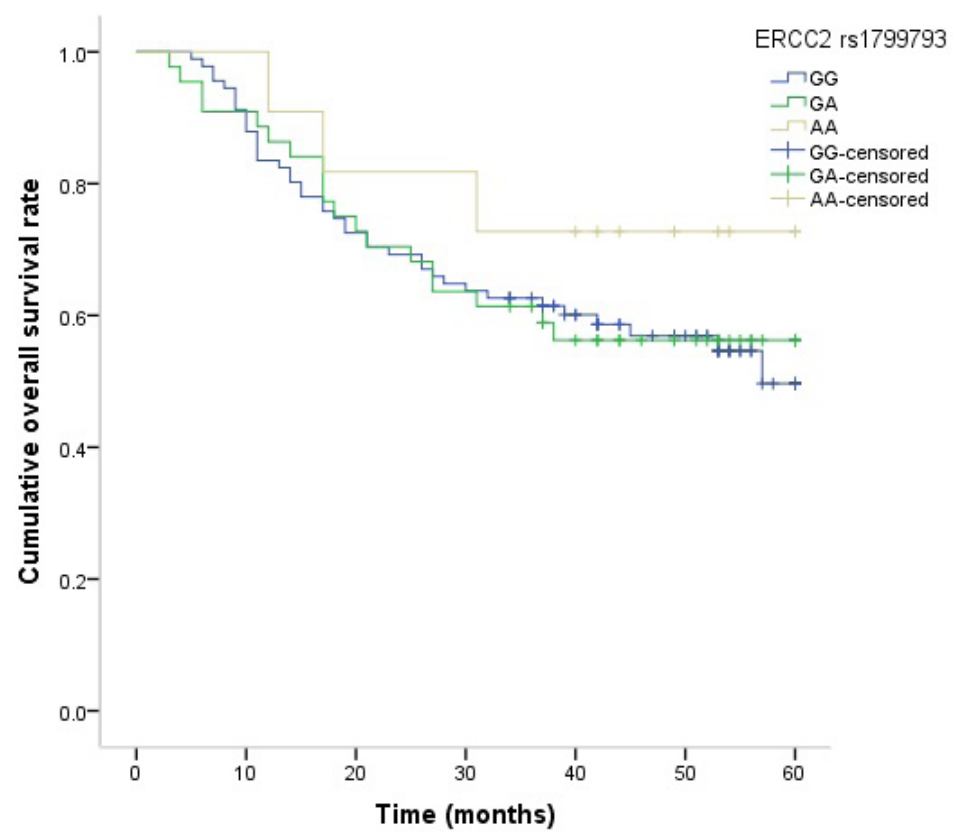

Figure 2. Kaplan-Meier survival curves showing relationship between ERCC2 rs1799793 polymorphisms and osteosarcoma patient survival.

\section{DISCUSSION}

DNA damage induced by several exogenous or endogenous factors requires efficient DNA repair to restore genomic integrity and involves a number of DNA repair genes. In our study, we found that polymorphisms in ERCC1 rs11615 and ERCC2 rs1799793 were associated with a pronounced reduced risk of death from osteosarcoma. ERCC1 and ERCC2 have critical functions in the NER mechanism. Our findings suggest that ERCC1 rs11615 and ERCC2 rs1799793 may be useful genetic prognostic markers for osteosarcoma and may be used as biomarkers for individualized therapy and post-operation treatment for different patients.

Previous studies reported that the ERCC1 and ERCC2 were associated with the prognosis of several types of cancers, such as colorectal, gastric, non-small cell lung, and bone cancers (Huang et al., 2013; Li et al., 2012, 2013, 2014; Yang and Xian, 2014). Li et al. (2012) examined 335 colorectal cancer patients and showed that ERCC1 rs11615 and ERCC2 rs1799793 genotyping may facilitate tailored oxaliplatin-based chemotherapy for colorectal cancer patients. In another study of 360 gastric cancer patients, it was found that the ERCC2 rs1799793 gene polymorphism may affect the overall survival of gastric cancer patients (Li et al., 2013). Yang and Xian (2014) conducted a meta-analysis of 46 studies including 9407 non-small cell lung cancer patients and reported that the ERCC1 rs11615 and ERCC2 rs13181 and rs1799793 gene polymorphisms may be useful as biomarkers for predicting the clinical outcomes of the platinum-based chemotherapy in these patients. Previous studies reported that NER pathway gene polymorphisms may effectively predict cancer prognosis.

For the association between ERCC1 and ERCC2 polymorphisms and the prognosis of patients with osteosarcoma, several previous studies reported the role of common variations in 
ERCC1 and ERCC2 on the clinical outcome of osteosarcoma (Biason et al., 2012; Hao et al., 2012; Li et al., 2014). Biason et al. (2012) investigated the role of NER gene variants in the prognosis of osteosarcoma and found that ERCC2 rs1799793 may be a prognostic marker of osteosarcoma. Hao et al. (2012) conducted a cohort study of 267 osteosarcoma patients and reported that ERCC1 rs11615 and ERCC2 rs1799793 influenced the clinical outcome of osteosarcoma patients. Li et al. (2014) conducted a meta-analysis and reported that the ERCC2 rs13181 polymorphism was associated with the overall survival of osteosarcoma patients. The discrepancies in these results may have been caused by differences in ethnicities, study design, and sample size, as well as by chance.

In conclusion, our results suggest that ERCC1 rs11615 and ERCC2 rs1799793 may be useful genetic prognostic markers for osteosarcoma in a Chinese population. Further multicenter studies involving various populations are needed to confirm our results.

\section{Conflicts of interest}

The authors declare no conflict of interest.

\section{REFERENCES}

Bai SB, Chen HX, Bao YX, Luo X, et al. (2013). Predictive impact of common variations in DNA repair genes on clinical outcome of osteosarcoma. Asian Pac. J. Cancer Prev. 14: 3677-3680.

Biason P, Hattinger CM, Innocenti F, Talamini R, et al (2012). Nucleotide excision repair gene variants and association with survival in osteosarcoma patients treated with neoadjuvant chemotherapy. Pharmacogenomics J. 12: 476-483.

Goode EL, Ulrich CM and Potter JD (2002). Polymorphisms in DNA repair genes and associations with cancer risk. Cancer Epidemiol. Biomarkers Prev. 11: 1513-1530.

Goričar K, Kovač V, Jazbec J, Zakotnik B, et al. (2015). Genetic variability of DNA repair mechanisms and glutathione-Stransferase genes influences treatment outcome in osteosarcoma. Cancer Epidemiol. 39: 182-188.

Hao T, Feng W, Zhang J, Sun YJ, et al. (2012). Association of four ERCC1 and ERCC2 SNPs with survival of bone tumour patients. Asian Pac. J. Cancer Prev. 13: 3821-3824.

He J, Wang J, Wang D, Dai S, et al. (2014). Association between CTLA-4 genetic polymorphisms and susceptibility to osteosarcoma in Chinese Han population. Endocrine 45: 325-330.

Huang MY, Tsai HL, Lin CH, Huang CW, et al. (2013). Predictive value of ERCC1, ERCC2, and XRCC1 overexpression for stage III colorectal cancer patients receiving FOLFOX-4 adjuvant chemotherapy. J. Surg. Oncol. 108: 457-464.

Li HY, Ge X, Huang GM, Li KY, et al. (2012). GSTP1, ERCC1 and ERCC2 polymorphisms, expression and clinical outcome of oxaliplatin-based adjuvant chemotherapy in colorectal cancer in Chinese population. Asian Pac. J. Cancer Prev. 13: 3465-3469.

Li J, Liu S, Wang W, Zhang K, et al. (2014). ERCC polymorphisms and prognosis of patients with osteosarcoma. Tumour Biol. 35: 10129-10136.

Li Y, Liu Z, Liu H, Wang LE, et al. (2013). ERCC1 and ERCC2 variants predict survival in gastric cancer patients. PLoS One 8: e71994.

Liu S, Yi Z, Ling M, Shi J, et al. (2014). Predictive potential of ABCB1, ABCC3, and GSTP1 gene polymorphisms on osteosarcoma survival after chemotherapy. Tumour Biol. 35: 9897-9904.

Mirabello L, Troisi RJ and Savage SA (2009). Osteosarcoma incidence and survival rates from 1973 to 2004: Data from the Surveillance, Epidemiology, and End Results Program. Cancer 115: 1531-1543.

Nouspikel T (2009). DNA repair in mammalian cells: Nucleotide excision repair: variations on versatility. Cell. Mol. Life Sci. 66: 994-1009.

Ottaviani G and Jaffe N (2009). The epidemiology of osteosarcoma. Cancer Treat. Res. 152: 3-13.

Picci P (2007). Osteosarcoma (osteogenic sarcoma). Orphanet journal of rare diseases. 2: 6.

Yang $Y$ and Xian $L$ (2014). The association between the ERCC1/2 polymorphisms and the clinical outcomes of the platinumbased chemotherapy in non-small cell lung cancer (NSCLC): a systematic review and meta-analysis. Tumour Biol. 35 : 2905-2921. 